\title{
Exploring New Multimodal Quantitative Imaging Indices for the Assessment of Osseous Tumor Burden in Prostate Cancer Using ${ }^{68}$ Ga-PSMA PET/CT
}

\author{
Marie Bieth ${ }^{* 1,2}$, Markus Krönke*1, Robert Tauber ${ }^{3}$, Marielena Dahlbender ${ }^{3}$, Margitta Retz ${ }^{3}$, Stephan G. Nekolla ${ }^{1}$, \\ Bjoern Menze ${ }^{2}$, Tobias Maurer ${ }^{3}$, Matthias Eiber $^{\dagger 1,4}$, and Markus Schwaiger ${ }^{\dagger 1}$ \\ ${ }^{I}$ Department of Nuclear Medicine, Klinikum Rechts der Isar, Technical University of Munich, Munich, Germany; ${ }^{2}$ Department of \\ Informatics, Technical University of Munich, Munich, Germany; ${ }^{3}$ Department of Urology, Klinikum Rechts der Isar, Technical \\ University of Munich, Munich, Germany; and ${ }^{4}$ Department of Molecular and Medical Pharmacology, David Geffen School of \\ Medicine at UCLA, University of California, Los Angeles, California
}

PET combined with CT and prostate-specific membrane antigen (PSMA) ligands has gained significant interest for staging prostate cancer (PC). In this study, we propose 2 multimodal quantitative indices as imaging biomarkers for the assessment of osseous tumor burden using ${ }^{68} \mathrm{Ga}-\mathrm{PSMA}$ PET/CT and present preliminary clinical data. Methods: We defined 2 bone PET indices (BPIs) that incorporate anatomic information from CT and functional information from ${ }^{68} \mathrm{Ga}-\mathrm{PSMA}$ PET: $\mathrm{BPI} \mathrm{IOL}_{\mathrm{V}}$ is the percentage of bone volume affected by tumor and $\mathrm{BPI}_{\mathrm{Suv}}$ additionally considers the level of PSMA expression. We describe a semiautomatic computation method based on segmentation of bones in CT and of lesions in PET. Data from 45 patients with castration-resistant PC and bone metastases during ${ }^{223} \mathrm{Ra}$-dichloride were retrospectively analyzed. We evaluated the computational stability and reproducibility of the proposed indices and explored their relation to the prostate-specific antigen blood value, the bone scan index (BSI), and disease classification using PERCIST. Results: On the technical side, BPIVOL and $\mathrm{BPI}_{\text {Suv }}$ showed an interobserver maximum difference of $3.5 \%$, and their computation took only a few minutes. On the clinical side, $\mathrm{BPI}_{\text {VOL }}$ and $\mathrm{BPI}_{\text {Suv }}$ showed significant correlations with $\mathrm{BSI}$ $(r=0.76$ and 0.74 , respectively, $P<0.001)$ and prostate-specific antigen values $(r=0.57$ and 0.54 , respectively, $P<0.01)$. When the proposed indices were compared against expert rating using PERCIST, BPIVOL and $\mathrm{BPI}_{\text {SUV }}$ showed better agreement than BSI, indicating their potential for objective response evaluation. Conclusion: We propose the evaluation of $\mathrm{BPI}_{\mathrm{VOL}}$ and $\mathrm{BPI}_{\mathrm{SuV}}$ as imaging biomarkers for ${ }^{68} \mathrm{Ga}-\mathrm{PSMA} \mathrm{PET} / \mathrm{CT}$ in a prospective study exploring their potential for outcome prediction in patients with bone metastases from PC.

Key Words: image processing; PET/CT; imaging biomarker; prostate cancer

J Nucl Med 2017; 58:1632-1637

DOI: 10.2967/jnumed.116.189050

Received Jan. 3, 2017; revision accepted Apr. 13, 2017.

For correspondence or reprints contact: Matthias Eiber, Department of Nuclear Medicine, Klinikum Rechts der Isar der TU München, Ismaninger Strasse 22, 81675 Munich, Germany.

E-mail: matthias.eiber@tum.de

${ }^{*}$ Contributed equally to this work.

tContributed equally to this work.

Published online May 25, 2017.

COPYRIGHT @ 2017 by the Society of Nuclear Medicine and Molecular Imaging.
$\mathbf{P}$ can later develop recurrence with cancer spread to other structures, in particular the bones. Both for primary tumor and for recurrence, bone scintigraphy, CT, and PET/CT are commonly used for staging. For bone scintigraphy, the bone scan index (BSI) (1) and its automatic computation method (2) can be used for quantitative analysis. However, in the absence of SPECT or SPECT/CT, bone scintigraphy is a 2-dimensional modality that lacks detailed anatomic information, has suboptimal specificity, and cannot reveal lymph node and visceral metastases. Because of its high detection rates and superb specificity for PC lesions ${ }^{68} \mathrm{Ga}$-prostate-specific membrane antigen (PSMA) PET/CT is increasingly used for staging of bone metastases instead of bone scintigraphy $(3,4)$. So far, for cross-sectional imaging only qualitative to semiquantitative methods for assessment of tumor burden and response such as RECIST (5) for CT and PERCIST $(6,7)$ for PET/CT have been developed. Additional important limitations for PC staging are RECIST is suboptimal for bone metastases staging because osteoblastic metastases without extraosseous tumor involvement are mainly regarded as nonmeasurable and PERCIST has been evaluated only for ${ }^{18} \mathrm{~F}$-FDG PET, which is rarely used in these patients.

Therefore, a comprehensive quantitative imaging biomarker that is capable of measuring the whole-body tumor burden, exploring the potential of a ${ }^{68} \mathrm{Ga}$-PSMA, is an unmet clinical need. A first approach for the quantitative assessment of ${ }^{18} \mathrm{~F}$-fluoride PET has been developed by Etchebehere et al. (8). However, it is unimodal and neglects the anatomic information contained in the CT image, therefore impeding interpatient comparability by, for example, not taking into account differences in patient size.

In this work, our aim was to define multimodal quantitative imaging indices using hybrid information from a ${ }^{68} \mathrm{Ga}$-PSMA PET/ CT scan. In a first step toward full-body quantification, we focused on bone tumor burden. We also developed a method to compute the indices automatically with possible manual corrections, so that they can be easily implemented in clinical practice. In addition, we have performed preliminary clinical analyses applying this method to a retrospective cohort of PC patients with bone metastases who underwent ${ }^{68} \mathrm{Ga}$-PSMA PET/CT under ${ }^{223} \mathrm{Ra}$-dichloride and compared the results with a reference standard of clinical expert reading, BSI, and serum prostate-specific antigen (PSA). 


\section{MATERIALS AND METHODS}

\section{Bone PET Index (BPI)}

Definition. BSI is defined as the percentage of skeletal mass affected by tumor calculated on a bone scintigram (1). Because bone scintigraphy is intrinsically 2-dimensional and lacks detailed anatomic information, a standard weighting of bones is incorporated in the calculation. The commercial software EXINI Bone BSI (EXINI Diagnostics AB) allows for automated calculation of the BSI. Inspired by this definition, we defined 2 new multimodal imaging indices for PET/CT: $\mathrm{BPI}_{\mathrm{VOL}}$ is the percentage of bone volume (including bone marrow) affected by tumor and $\mathrm{BPI}_{\mathrm{SUV}}$ additionally considers the target expression measured by the average SUV. In both indices, the anatomic information was extracted from the CT image whereas the functional information was extracted from the PET image, making them intrinsically multimodal. They were calculated as follows, where $N$ is the number of pixels $p$ that belong to bone metastases:

$$
\begin{gathered}
\mathrm{BPI}_{\mathrm{VOL}}=100 \times \frac{\text { Bone metastases volume }}{\text { Skeleton volume }}(\text { no unit }) \\
\mathrm{SUV}_{\text {mean }}=\frac{1}{N} \sum_{\substack{p \text { in bone } \\
\text { metastases }}} \mathrm{SUV}(p)(\text { unit: } \mathrm{g} / \mathrm{mL}) \\
\mathrm{BPI}_{\mathrm{SUV}}=\mathrm{BPI}_{\mathrm{VOL}} \times \mathrm{SUV}_{\text {mean }} / 100(\text { unit: } \mathrm{g} / \mathrm{mL})
\end{gathered}
$$

Notably, BPI $_{\mathrm{VOL}}$ differs from the metabolic tumor volume and BPI $\mathrm{SUV}_{\mathrm{SU}}$ from the total lesion glycosis because of normalization by skeleton volume. Contrarily to the calculation of BSI, no standard weighting of the bones is needed because patient-specific anatomic information from the CT image is used instead. In PET/CT, depending on the type of cancer, regularly only the trunk and not the whole body is imaged. Therefore, arms and legs as well as part of the head were excluded from the computation. To achieve a standardized calculation of the BPI that accounted for variation in the field of view and allowed for inter- and intrapatient comparison, only the slices between the bottom of the ischium (easily recognized on CT) and the caudal edge of the sublingual gland (easily recognized because of glandular uptake in PET) were considered. Of note, in the computation of BSI by EXINI Bone ${ }^{\mathrm{BSI}}$, the forearms and lower legs are excluded as well.

Semiautomatic Computation Method. For the computation of $\mathrm{BPI}_{\mathrm{VOL}}, \mathrm{SUV}_{\text {mean }}$, and $\mathrm{BPI}_{\mathrm{SUV}}$, a precise segmentation of the skeleton in $\mathrm{CT}$ and of bone metastases in PET were necessary. These segmentations could have been done manually with appropriate software, but would have been time consuming. We proposed instead an automatic method with possible manual corrections that we have implemented using the programming language Python (9).

Our tool read images in DICOM format. PET and CT were affinely registered using the information contained in the DICOM headers. This was possible because both images were acquired on the same scanner during the same session. The bed was automatically removed from the $\mathrm{CT}$ by simple morphologic operations.

On CT, the skeleton can be segmented using its density in Hounsfield units, which is higher than that of soft tissue and air. Following the first 2 steps of the method of Kang et al. (10), we used global thresholding of the CT image followed by local adaptive thresholding to obtain a first bone mask. Then we applied a supplementary morphologic hole closing to obtain a segmentation of the bones and bone marrow. In the case of heavy calcification or in the case of artifact resulting, for example, from implants, manual

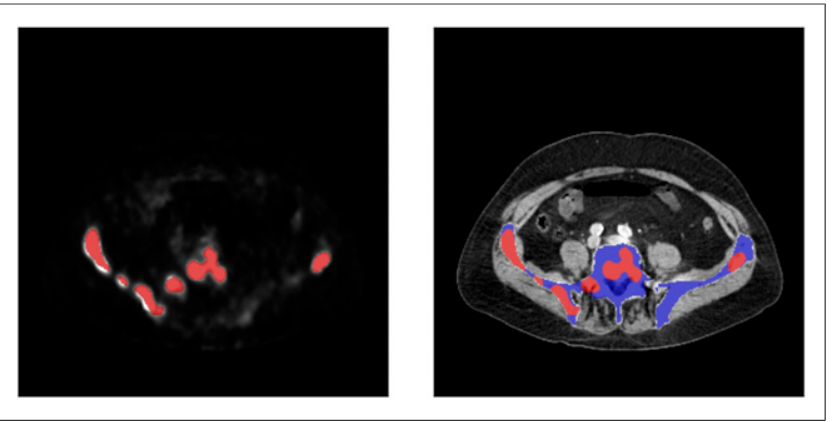

FIGURE 1. PET (left) and CT (right) image of 1 patient. Blue overlay shows bone mask computed by tool, and red overlay shows tumor mask computed with SUV threshold of 3.

corrections were possible: corrections could be applied either with a brush or by removing in 1 click the whole "bone" from a slice. Details on the computation method are given in the supplemental materials (available at http://jnm.snmjournals.org).

${ }^{68} \mathrm{Ga}$-PSMA usually does not exhibit unspecific uptake in the bones and bone marrow. Therefore, regions of the skeleton with increased uptake can be considered as bone metastases. Thus, the lesions were segmented using a SUV threshold on PET and restricting the result to the skeleton segmented from the CT image (Fig. 1). This use of anatomic information avoided manual removal of normal uptake sites (e.g., bladder, kidneys) as proposed by Etchebehere et al. (8). Even though CT and PET are acquired consecutively on the same scanner, for example, breathing can cause misalignment in the region of the ribs. Because the liver and spleen show high physiologic uptake of ${ }^{68} \mathrm{Ga}$-PSMA, projection of the ribs in CT on liver and spleen in PET can potentially generate false-positives. When our current tool was used, such false-positives had to be manually corrected.

The final segmentation depended on the SUV used to threshold the PET as described in the previous paragraph. Rather than choosing an arbitrary threshold, we therefore developed a method to choose the threshold based on the maximum tolerated false-positive rate using negative training patients. For each patient, the lowest SUV threshold resulting in the given false-positive rate was automatically computed. $\mathrm{BPI}_{\mathrm{VOL}}$ values obtained during this search are shown for 1 training patient in Figure 2. Using the maximum of the thresholds computed for all training patients ensured that the false-positive rate did not exceed the maximum for any training patient. The effects of choosing different maximum tolerated falsepositive rates on $\mathrm{BPI}_{\mathrm{VOL}}$ and $\mathrm{BPI}_{\mathrm{SUV}}$ are presented in the "Results" section.

\section{Patients Cohort}

Between January 2014 and February 2016, 45 patients with metastatic castration-resistant PC (mCRPC) with bone metastases but no organ or relevant lymph node $(>3 \mathrm{~cm})$ metastases underwent ${ }^{223} \mathrm{Ra}$-dichloride therapy (Xofigo; Bayer Healthcare) at our institution. The mean age of the patients was $71(\mathrm{SD}, \pm 8 \mathrm{y})$. All patients received therapy at a dose of $50 \mathrm{kBq} / \mathrm{kg}$ per therapy cycle in monthly intervals with up to 6 cycles. Fifteen patients who underwent a ${ }^{68} \mathrm{Ga}$-PSMA $\mathrm{PET} / \mathrm{CT}$ at our institution and were regarded as negative for bone metastases by an experienced nuclear medicine physician were also retrospectively randomly selected to serve as a negative training cohort for the method.

All patients underwent ${ }^{68} \mathrm{Ga}$-PSMA PET/CT within 4 wk before initiation of ${ }^{223}$ Ra-dichloride therapy. Thirty-one patients also underwent bone scintigraphy. Thirty-two patients underwent additional 


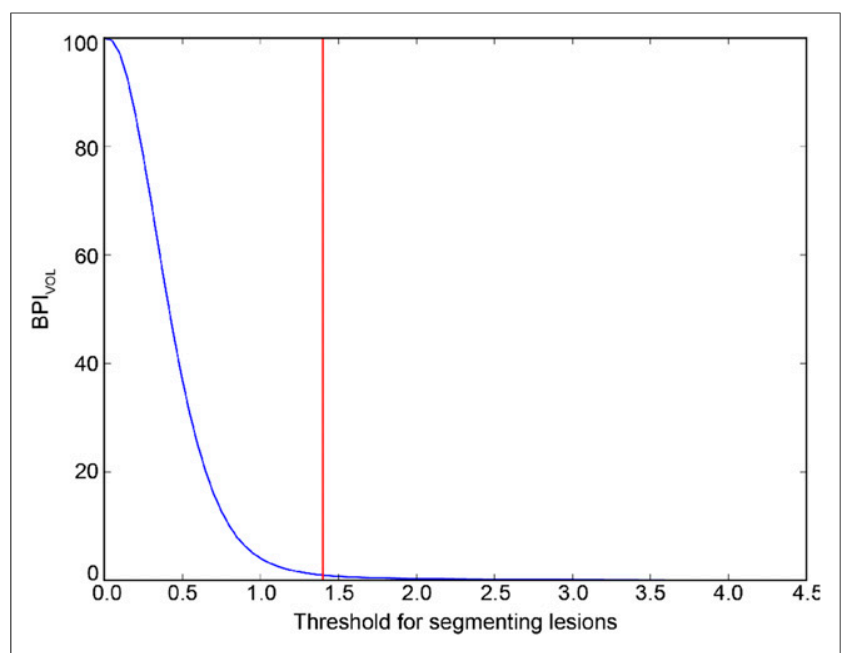

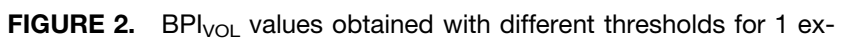
ample of negative training patient. Red line indicates $\mathrm{BPI}_{\mathrm{VOL}}$ under 1 are obtained for thresholds superior to 1.4 .

${ }^{68}$ Ga-PSMA PET/CT and 22 of them bone scintigraphy 3-6 mo after the first scan. Table 1 shows a summary of data available for all patients.

The institutional review board of the Technical University Munich approved the retrospective analysis (permit 5665/13), and all subjects signed a written informed consent form for anonymized evaluation and publication of their data.

\section{Data Acquisition}

${ }^{68}$ Ga-PSMA PET/CT was performed approximately $54 \mathrm{~min}$ ( $\pm 8 \mathrm{~min}$; range, $43-88 \mathrm{~min}$ ) after injection of a mean of $133 \mathrm{MBq}$ (379 MBq; range, 52-239 MBq) of ${ }^{68} \mathrm{Ga}$-labeled HBED-CC. A diagnostic CT scan was obtained in the portal venous phase after intravenous injection of contrast agent (Imeron 300; Bracco IMAGING Deutschland $\mathrm{GmbH}$ ). Immediately after the $\mathrm{CT}$, the PET scan was acquired with $6-8$ bed positions (3-5 min per bed position). PET was reconstructed using ordered-subset expectation-maximization with pointspread function and time-of-flight information (3 iterations, 21 subsets) and corrected for normalization, attenuation, scatter, randoms, and decay. The transaxial pixel size was $4.07 \mathrm{~mm}$ for PET and $1.52 \mathrm{~mm}$ for $\mathrm{CT}$, and the slice thickness was $5 \mathrm{~mm}$ for both. ${ }^{99 \mathrm{~m}} \mathrm{Tc}-\mathrm{HDP}$ whole-body bone scintigraphy was performed in planar imaging mode with an acquisition time of $1 \mathrm{~min} / 10 \mathrm{~cm}$ of body height. Activity was body weight-adjusted $(9 \mathrm{MBq} / \mathrm{kg})$ and injected $3 \mathrm{~h}$ before imaging.

\section{Data and Statistical Analysis}

To validate our new tool and the introduced BPI, we performed a reproducibility analysis. Randomly selected datasets of $10 \mathrm{mCRPC}$ patients before application of ${ }^{223} \mathrm{Ra}$-dichloride were analyzed by 2 trained observers applying manual corrections independently. The reproducibility threshold was then defined as the maximum absolute difference observed between both observers for each index. For BSI, Anand et al. defined the reproducibility threshold as 0.30 (11).

For response assessment, BPI was compared with BSI, PERCIST performed by an experienced reader, and PSA. BSI was computed from the bone scintigraphy images using the commercially available EXINI Bone ${ }^{\text {BSI }}$. Response by PERCIST was evaluated by an experienced physician using recently published criteria $(6,7)$, and criteria were adapted for ${ }^{68} \mathrm{Ga}$-PSMA similarly to a recent work (12). In brief, $\mathrm{SUV}_{\text {peak }}$ was measured in 1-5 target lesions and the appearance of new lesions was investigated.

Because PERCIST is not quantitative, but only classifies the patient as having progressive metabolic disease, stable metabolic disease, or partial metabolic response, we also defined these categories for BPI and BSI using the respective reproducibility threshold: a change of magnitude smaller than the reproducibility threshold was considered as stable metabolic disease, an increase in value larger than the reproducibility threshold was considered as progressive disease, and a decrease in value larger than the reproducibility threshold was considered as partial metabolic response. Moreover, 2 separate analyses based on PERCIST were performed: metastatic status based on all types of target lesions (including potential new lymph node and visceral metastases, as prescribed by the criteria) and metastatic status based on bone involvement only (to allow for direct comparison with BPI).

For comparing quantitative methods (i.e., BPI, BSI, and PSA), we used the Pearson coefficient $r$. For all tests, a $P$ value smaller than 0.05 was considered significant.

\section{RESULTS}

\section{Technical Validation}

Bone Segmentation and Manual Corrections. After manual correction, the mean bone volume of the 45 treated patients was $4,184 \mathrm{~cm}^{3}\left( \pm 503 \mathrm{~cm}^{3}\right.$; range, 3,327-5,739 $\left.\mathrm{cm}^{3}\right)$. For 32 patients with 2 sequential ${ }^{68} \mathrm{Ga}$-PSMA PET/CT scans, the mean difference in computed bone volume between 2 scans was $66 \mathrm{~cm}^{3}( \pm 61$ $\mathrm{cm}^{3}$ ), with a maximum of $270 \mathrm{~cm}^{3}$. After bones had been segmented and manually corrected, false-positives (e.g., in the rib cage) were corrected by an expert reader. On 54 scans from the patient cohort, an average of $3.8 \mathrm{~cm}^{3}$ false-positives per patient had to be manually corrected. This represents an average difference in $\mathrm{BPI}_{\mathrm{VOL}}$ of 0.0008 per patient.

Selection of SUV Threshold for Lesion Segmentation. The SUV threshold lesion segmentation was determined using 15 negative training patients. For each patient, we computed the threshold that resulted in a BPI $\mathrm{VOL}$ of 0.1 and 1 (equal to $0.1 \%$ and $1 \%$ of falsepositive voxels), respectively. Corresponding SUV thresholds for all negative training patients were in the range of 1.15-1.95 (mean, 1.42) for a false-positive threshold of 1 and a range of 1.7-2.65 (mean, 2.06) for 0.1 , respectively. Figure 2 shows the different $\mathrm{BPI}_{\mathrm{VOL}}$ values obtained for different thresholds for 1 negative training patient.

TABLE 1

Available Data for Total of 60 Patients

\begin{tabular}{|c|c|c|c|c|}
\hline Parameter & Test & Before therapy & After 3 cycles & After 6 cycles \\
\hline Negative training patients & ${ }^{68} \mathrm{Ga}-\mathrm{PSMA} \mathrm{PET} / \mathrm{CT}$ & 15 & Not applicable & Not applicable \\
\hline \multirow[t]{3}{*}{ Patients undergoing ${ }^{223} \mathrm{Ra}$-dichloride therapy } & ${ }^{68} \mathrm{Ga}-\mathrm{PSMA} \mathrm{PET} / \mathrm{CT}$ & 45 & 32 & 20 \\
\hline & PSA value & 43 & 33 & 21 \\
\hline & Bone scan & 31 & 21 & 18 \\
\hline
\end{tabular}




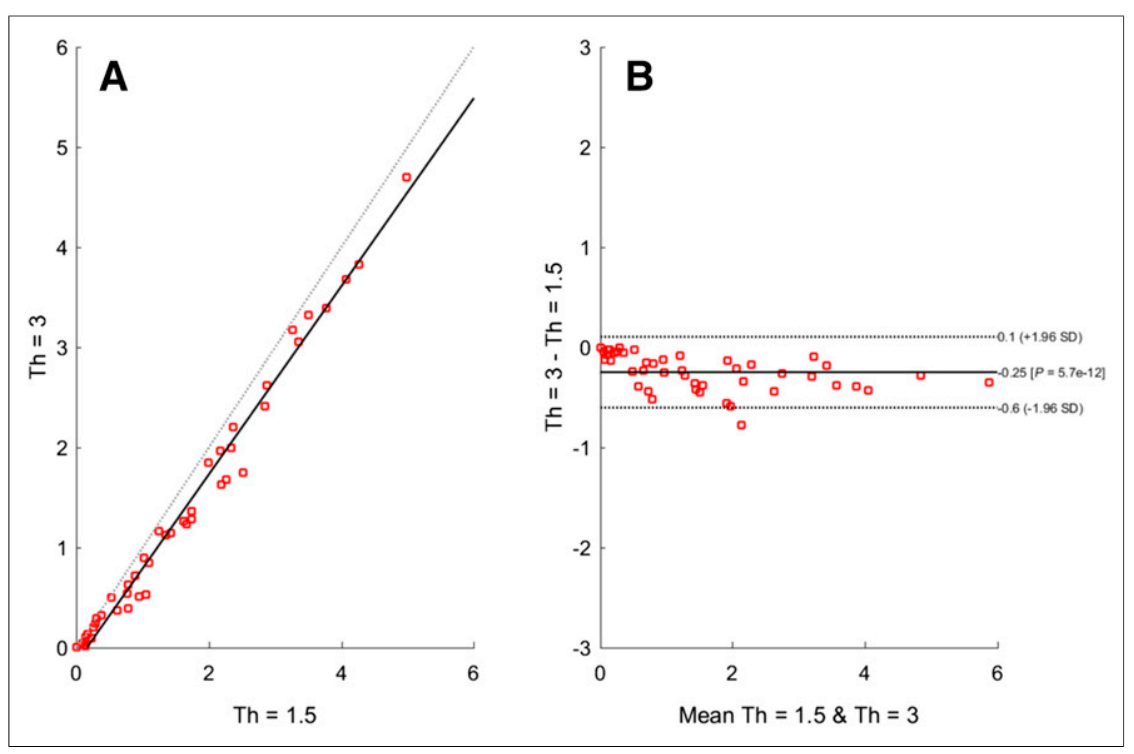

FIGURE 3. Comparison between BPI $\mathrm{BUV}_{\mathrm{Su}}$ obtained with cutoff values 1.5 and 3 . High correlation between results is shown both using a scatter diagram (A) and in the Bland-Altman plot on which differences between $2 \mathrm{BPI}_{\text {SUV }}$ are plotted against their average (B). As expected, it shows mean difference of $-0.25 \mathrm{BPI}_{\text {suv }}\left(95 \%\right.$ confidence intervals, +0.1 and $-0.6 \mathrm{BPI}_{\text {suv }}$ ), indicating systematically lower $\mathrm{BPI}_{\mathrm{Suv}}$ values for a cutoff value of 3 .

SUV Threshold Influence on Lesion Segmentation. On the basis of these results, thresholds of 1.5 (mean value obtained for the scenario of $1 \%$ false-positive results) and 3 (conservative approach ensuring a maximum of $0.1 \%$ false-positive lesions in all patients) were used for the initial analysis of the baseline ${ }^{68} \mathrm{Ga}-\mathrm{PSMA}$ PET/ CT of all 45 patients. There was a strong correlation between the

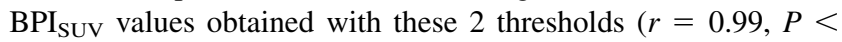
0.001; Fig. 3). This also held for $\mathrm{BPI}_{\mathrm{VOL}}$ and $\mathrm{SUV}_{\text {mean }}(r=0.95$ for both, $P<0.001)$. Because of the high correlation between both values, we chose a threshold of 3 to ensure a high specificity of the BPI, with less than 0.1 of $\mathrm{BPI}_{\mathrm{VOL}}$ being related to falsepositives. All following results were computed using a SUV threshold of 3 .

Reproducibility. Comparison from 2 independent observers using 10 randomly selected datasets showed a nearly perfect correlation ( $r=0.999, P<0.001$ for both). The maximum observed percentage differences between both observers were $3.5 \%$ for $\mathrm{BPI}_{\mathrm{SUV}}$ and $2.2 \%$ for $\mathrm{BPI}_{\mathrm{VOL}}$. The maximum absolute difference was 0.055 for $P=0.32$ ).
BPI $_{\mathrm{SUV}}$ and 0.37 for BPI $\mathrm{VOL}_{\mathrm{VO}}$. Rounding up these values, we defined 0.06 and 0.4 as reproducibility thresholds for the respective index. Note that a wide range of disease was present in the analyzed patients (range, BPI $\mathrm{BUV}_{\mathrm{SUV}}$ 0.09-3.39, and $\mathrm{BPI}_{\mathrm{VOL}}$ : 1.53-38.05).

\section{Quantification Using BPIVoL, SUV $_{\text {mean, }}$ and $\mathrm{BPI}_{\text {suv }}$}

$B P I_{V O L}, S U V_{\text {mean }}$, and $B P I_{S U V}$ Before and After Therapy. The average values of $\mathrm{BPI}_{\mathrm{VOL}}, \mathrm{SUV}_{\text {mean }}$, and $\mathrm{BPI}_{\mathrm{SUV}}$ before therapy were $19.5,8.3$, and 1.59 , respectively. After therapy, the average values were 26.0, 7.7, and 1.99. These values represented changes of $+33 \%,-7 \%$, and $+25 \%$, respectively.

Correlation Between $B P I_{S U V}$ and $B P I_{V O L}$. $\mathrm{BPI}_{\mathrm{SUV}}$ and $\mathrm{BPI}_{\mathrm{VOL}}$ for all $45 \mathrm{mCRPC}$ patients before therapy were strongly correlated ( $r=0.89, P<0.001$; Fig. 4A). The percentage changes of $\mathrm{BPI}_{\mathrm{VOL}}$ and $\mathrm{BPI}_{\mathrm{SUV}}$ during therapy were strongly correlated ( $r=0.97, P<0.001$; Fig. 4B).

\section{Correlation of BPI to Clinical Parameters}

At baseline, $\mathrm{BPI}_{\mathrm{VOL}}$ and $\mathrm{BPI}_{\mathrm{SUV}}$ showed a moderate and significant correlation with BSI ( $r=0.76$ and 0.74 , respectively, $P<$ 0.001; Fig. 4C). There was a tendency to a stronger correlation with PSA value for $\mathrm{BPI}_{\mathrm{VOL}}$ and $\mathrm{BPI}_{\mathrm{SUV}}(r=0.57$ and 0.54 , respectively, $P<0.01)$ than for BSI $(r=0.49, P<0.01)$.

A moderate correlation between change of $\mathrm{BPI}_{\mathrm{VOL}}$ and $\mathrm{BPI}_{\mathrm{SUV}}$ and percentage change of PSA value after treatment was observed $(r=0.70, P<0.01)$. There was no correlation of change in BSI with percentage change in PSA value $(r=0.24$,

When compared with PERCIST for all type of target lesions (Table 2), BPI $\mathrm{VOL}_{\mathrm{VO}}, \mathrm{BPI}_{\mathrm{SUV}}$, and BSI showed agreement for $65.6 \%$ $(21 / 32), 68.7 \%(22 / 32)$, and $57.9 \%(11 / 19)$ of patients and opposite results for $25.0 \%(8 / 32), 15.6 \%(5 / 32)$, and $21.1 \%(4 / 19)$, respectively. When compared with PERCIST for bone involvement only (Table 2), BPI $\mathrm{VOL}_{\mathrm{VO}}, \mathrm{BPI}_{\mathrm{SUV}}$, and BSI showed agreement for $62.5 \%$ (20/32), $68.7 \%$ (22/32), and $63.2 \%$ (12/19) of patients
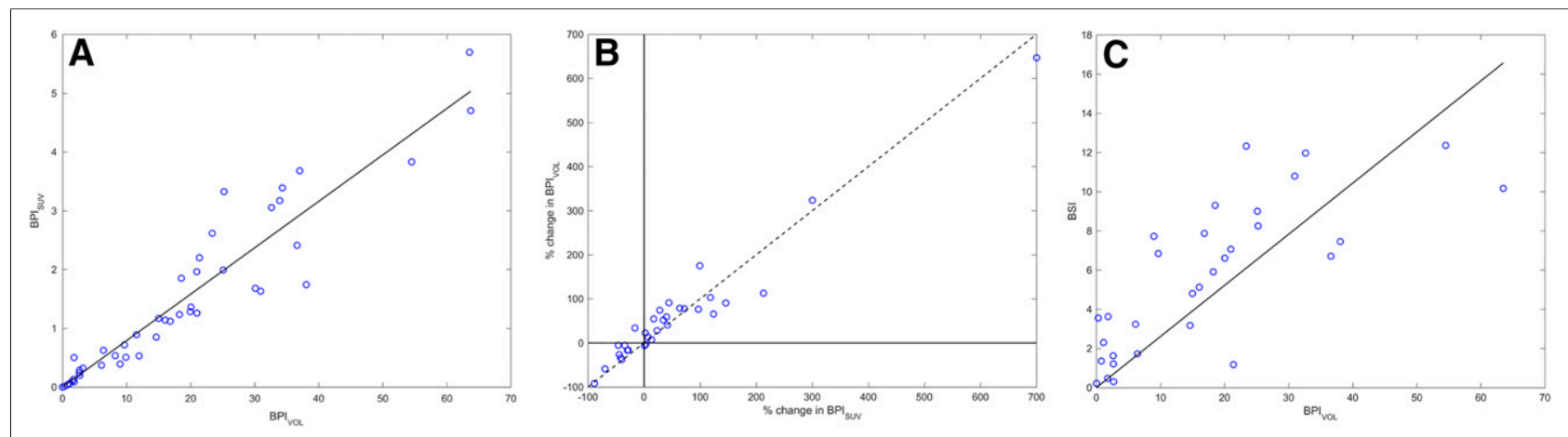

FIGURE 4. (A) BPI $\mathrm{VOL}_{\mathrm{V}}$ and $\mathrm{BPI}_{\mathrm{SUV}}$ values. Statistically significant high correlation $(r=0.89, P<0.001)$ was observed. Images from all 45 patients with bone metastases were used. (B) Change in $\mathrm{BPI}_{\mathrm{SuV}}$ and $\mathrm{BP} \mathrm{V}_{\mathrm{VOL}}$ during therapy for 32 patients. Statistically significant high linear correlation $(r=0.97, P<$ 0.001) was observed. (C) BPI VOL and BSI values for 31 patients before beginning of therapy. Moderate correlation $(r=0.76, P<0.001)$ was observed. 
TABLE 2

Comparison of Expert Reader and BPI and BSI Classifications

\begin{tabular}{|c|c|c|c|c|c|c|c|c|c|c|}
\hline \multirow[b]{2}{*}{ Parameter } & \multirow[b]{2}{*}{ Classification } & \multicolumn{3}{|c|}{$\mathrm{BPI}_{\mathrm{VOL}}$} & \multicolumn{3}{|c|}{$\mathrm{BPI}_{\text {Suv }}$} & \multicolumn{3}{|c|}{$\mathrm{BSI}$} \\
\hline & & Prog & Stab & Resp & Prog & Stab & Resp & Prog & Stab & Resp \\
\hline \multirow[t]{3}{*}{ PERCIST } & Prog & 17 & 2 & 6 & 17 & 3 & 5 & 8 & 4 & 4 \\
\hline & Stab & 1 & 0 & 0 & 1 & 0 & 0 & 0 & 1 & 0 \\
\hline & Resp & 2 & 0 & 4 & 0 & 1 & 5 & 0 & 0 & 2 \\
\hline \multirow[t]{3}{*}{ Bone PERCIST } & Prog & 15 & 2 & 2 & 15 & 2 & 2 & 7 & 2 & 2 \\
\hline & Stab & 3 & 0 & 3 & 3 & 1 & 2 & 1 & 3 & 2 \\
\hline & Resp & 2 & 0 & 5 & 0 & 1 & 6 & 0 & 0 & 2 \\
\hline
\end{tabular}

and opposite results for $12.5 \%$ (4/32), $6.2 \%$ (2/32), and 10.5\% (2/19), respectively. An exemplary case of a patient showing divergent results with considerable decrease of PSMA expression under therapy indicating response in $\mathrm{BPI}_{\mathrm{VOL}}$ and $\mathrm{BPI}_{\mathrm{SUV}}$ but progressive disease due to new lesions in PERCIST is given in Figure 5. Notably, cases in which results from one method indicated stable disease but the other method indicated progression or response were not counted as opposite results.

\section{DISCUSSION}

In this study, we have described the BPIs as new quantitative multimodal imaging indices for the assessment of bone metastases in ${ }^{68} \mathrm{Ga}$-PSMA PET/CT using an automatic computation method. We have shown that, on our patient cohort, small differences in SUV thresholds and small misalignments between PET and CT did not substantially influence the results. Different observers also obtained similar values of the indices, showing a good interobserver reproducibility. A small amount of manual correction was still necessary, especially because of calcifications and endoprothesis.

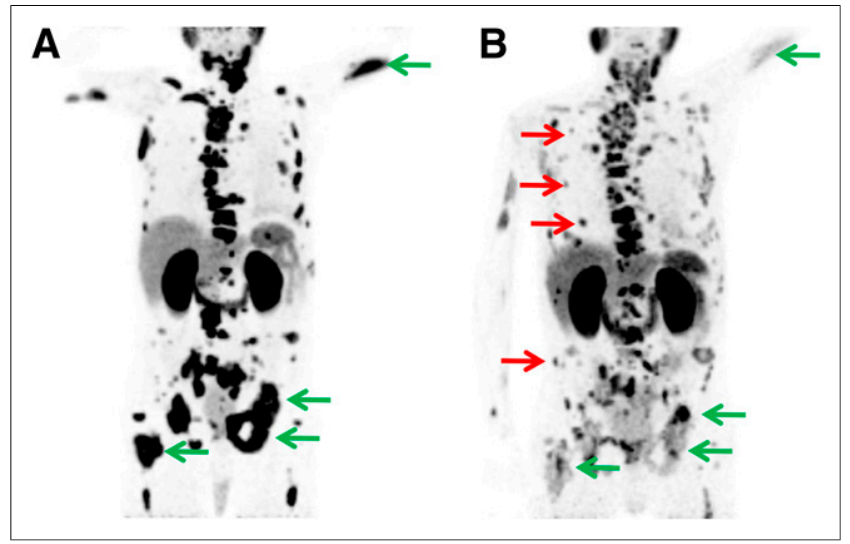

FIGURE 5. Maximum-intensity projection of ${ }^{68} \mathrm{Ga}$-PSMA PET images of same patient before (A) and after (B) 3 cycles of ${ }^{223}$ Ra-dichloride therapy. Most bone lesions show substantial response to therapy based on decreasing PSMA expression (green arrows), but also a few new lesions (red arrows) appear on follow-up scan. Despite an overall decrease in tumor load, based on PERCIST status of patient was defined as progressive disease.
The computation of both indices with our software took only a few minutes, including manual corrections of skeleton segmentation and false-positives. In contrast, a complete manual segmentation of a whole-body dataset would take several hours even for an experienced physician, because the skeleton and not only the lesions have to be segmented.

From a clinical perspective, our preliminary data indicated that BPI holds potential for quantitative response assessment. This was documented by a high correlation of BPI with BSI and PSA in mCRPC patients, and reasonable prediction of tumor response compared with PERCIST. We are aware that these results allow only for a first estimation of the potential clinical usability due to the known limitations of theses comparators. Therefore, future prospective clinical studies using more objective endpoints (overall survival, radiographic progression-free survival, skeletal adverse events) are necessary to fully investigate the potential of the proposed quantitative biomarkers for response prediction.

Compared with the imaging biomarkers $\mathrm{FTV}_{10}$, representing the volume of fluoride bone metastases, and $\mathrm{TLF}_{10}$, representing the total fluoride metastatic uptake introduced by Etchebehere et al. (8), calculation of $\mathrm{BPI}_{\mathrm{VOL}}$ and $\mathrm{BPI}_{\mathrm{SUV}}$ was an intrinsically multimodal approach. $\mathrm{TLF}_{10}$ and $\mathrm{FTV}_{10}$ exclusively rely on the information from PET, and no correction is possible with regard to the patient size. In contrast, for $\mathrm{BPI}_{\mathrm{VOL}}$ and $\mathrm{BPI}_{\mathrm{SUV}}$, the skeleton volume based on additional information from CT was included as well, thus allowing for interpatient comparison.

In a first step, we demonstrated that the bone volume using the newly introduced tool was reproducible between different scans of the same patient (mean difference, $<2 \%$ ). The small discrepancy can be explained by slightly different positions of the patient in the scanner. The absolute values obtained in our study (mean, 4,184 \pm $503 \mathrm{~cm}^{3}$ ) were in the expected range of a reference human skeleton, with an estimated total volume (including bone marrow, averaged for both sexes) of $7,700 \mathrm{~cm}^{3}$ (13), of which an average of $50.3 \%$ was included in our segmentation (14).

In a second step, both imaging indices $\mathrm{BPI}_{\mathrm{VOL}}$ and $\mathrm{BPI}_{\mathrm{SUV}}$ proved highly reproducible, with a maximum interobserver difference of $3.5 \%$. From a practical point of view, the false-positive correction was done with only a few clicks and the full image segmentation with manual corrections took only a few minutes, which makes it usable in clinical practice. The limited influence of different SUV thresholds for computation underlined the 
robustness for clinical analyses. For the quantitative read-out of both indices, we finally chose a conservative approach (SUV cutoff, 3) to ensure that less than 0.1 is due to false-positives.

In principle, the 2 introduced indices $\mathrm{BPI}_{\mathrm{VOL}}$ and $\mathrm{BPI}_{\mathrm{SUV}}$ were highly correlated. Nevertheless, it is important to mention that the information they provide is not completely equivalent, because BPI $_{S U V}$ also took into account the level of expression of PSMA. The percentage changes of $\mathrm{BPI}_{\mathrm{VOL}}$ and $\mathrm{BPI}_{\mathrm{SUV}}$ during therapy were highly correlated. Interestingly, whereas the average values of $\mathrm{BPI}_{\mathrm{VOL}}$ and $\mathrm{BPI}_{\mathrm{SUV}}$ increased during therapy, the average value of $\mathrm{SUV}_{\text {mean }}$ decreased, showing that $\mathrm{BPI}_{\mathrm{VOL}}, \mathrm{SUV}_{\text {mean }}$, and BPI $_{\text {SUV }}$ provided different information.

Notably, some clear outliers in the comparison between BPI and BSI (Fig. 4C) were observed. It has to be respected that bone scintigraphy and ${ }^{68} \mathrm{Ga}$-PSMA PET/CT image 2 different biologic processes: bone scintigraphy displays the reactive changes of the tumor on the skeleton (15), whereas ${ }^{68}$ Ga-PSMA PET directly shows the intensity of PSMA expression on viable tumor cells. Thus, no absolute equivalence of BSI and BPI could be expected. Other explaining factors were the higher sensitivity of ${ }^{68} \mathrm{Ga}$-PSMA PET/CT for bone metastases (16), the delay after which bone scintigraphy usually shows changes as well as the flare phenomenon $(15,17)$.

Compared with standardized imaging response evaluation using PERCIST results for all types of target lesion, $\mathrm{BPI}_{\text {SUV }}$ showed a

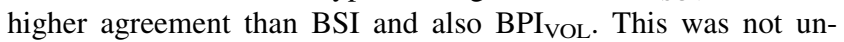
expected because $\mathrm{BPI}_{\mathrm{SUV}}$ considers also quantitative values, as does PERCIST. An even better correlation with a lower number of patients showing divergent results between PERCIST and both indices was achieved when they were compared with PERCIST results based only on bone involvement. This clearly implied the need of further adaptation of BPI as a quantitative PET imaging index also for the assessment soft-tissue tumor burden. A further argument for the potential clinical value of BPI compared with conventional methods was the statistically significant substantial correlation of BPI with percentage change of PSA value during therapy $(r=0.70, P<0.01)$ compared with BSI, which showed no correlation $(r=0.24, P=0.32)$.

Our study had several limitations. First, the study was based on retrospective data analysis. Second, after the baseline scan before ${ }^{223}$ Ra-dichloride follow-up scans for assessment of therapy response were either after 3 or after 6 cycles. Nevertheless, the aim of the study was primarily the introduction of a new quantitative imaging biomarker and only second to establish first preliminary correlation to clinical data. The influence of previous lines of treatment was not assessed, which could potentially affect signals derived from both ${ }^{68} \mathrm{Ga}$-PSMA PET and bone scanning $(18,19)$. These confounding factors should be investigated in future studies encompassing larger patient cohorts. Another limitation is the fact that no respiratory gating was used, which potentially would minimize the need for manual correction.

\section{CONCLUSION}

We have introduced $\mathrm{BPI}_{\mathrm{SUV}}$ and $\mathrm{BPI}_{\mathrm{VOL}}$ as new multimodal quantitative imaging indices for ${ }^{68} \mathrm{Ga}$-PSMA PET/CT, representing a robust tool for quantitative assessment of osseous tumor burden. We have shown that their automatic computation (with minimal manual corrections) is feasible and highly reproducible on a retrospective cohort of mCRPC patients. Finally, our results demonstrated that $\mathrm{BPI}_{\mathrm{VOL}}$ and $\mathrm{BPI}_{\mathrm{SUV}}$ provide clinically meaningful information when correlated to PERCIST, BSI, and PSA value.
However, the full clinical value, for example, predicting patient outcome, has to be investigated in future prospective studies.

\section{DISCLOSURE}

This work was partially funded by the German Ministry for Education and Research (Bundesministerium für Bildung und Forschung) under grant agreement no. 01IS12057, by the European Union Seventh Framework Program (FP7) under grant agreement no. 294582 ERC MUMI, and by the German Research Society (DFG) under grant agreement no. SFB 824. No other potential conflict of interest relevant to this article was reported.

\section{REFERENCES}

1. Imbriaco M, Larson SM, Yeung HW, et al. A new parameter for measuring metastatic bone involvement by prostate cancer: the Bone Scan Index. Clin Cancer Res. 1998;4:1765-1772.

2. Ulmert D, Kaboteh R, Fox JJ, et al. A novel automated platform for quantifying the extent of skeletal tumour involvement in prostate cancer patients using the bone scan index. Eur Urol. 2012;62:78-84.

3. Afshar-Oromieh A, Malcher A, Eder M, et al. PET imaging with a $\left[{ }^{68} \mathrm{Ga}\right]$ galliumlabelled PSMA ligand for the diagnosis of prostate cancer: biodistribution in humans and first evaluation of tumour lesions. Eur J Nucl Med Mol Imaging. 2013;40:486-495.

4. Afshar-Oromieh A, Zechmann CM, Malcher A, et al. Comparison of PET imaging with a ${ }^{68} \mathrm{Ga}$-labelled PSMA ligand and ${ }^{18} \mathrm{~F}$-choline-based PET/CT for the diagnosis of recurrent prostate cancer. Eur J Nucl Med Mol Imaging. 2014;41:11-20.

5. Eisenhauer EA, Therasse P, Bogaerts $J$, et al. New response evaluation criteria in solid tumours: revised RECIST guideline (version 1.1). Eur $J$ Cancer. 2009; 45:228-247.

6. Wahl RL, Jacene H, Kasamon Y, Lodge MA. From RECIST to PERCIST: evolving considerations for PET response criteria in solid tumors. J Nucl Med. 2009;50(suppl 1):122S-150S.

7. O JH, Lodge MA, Wahl RL. Practical PERCIST: a simplified guide to PET response criteria in solid tumors 1.0. Radiology. 2016;280:576-584.

8. Etchebehere EC, Araujo JC, Fox PS, Swanston NM, Macapinlac HA, Rohren EM. Prognostic factors in patients treated with ${ }^{223} \mathrm{Ra}$ : the role of skeletal tumor burden on baseline ${ }^{18} \mathrm{~F}$-fluoride $\mathrm{PET} / \mathrm{CT}$ in predicting overall survival. J Nucl Med. 2015;56:1177-1184.

9. Python Language Reference, version 2.7. Python Software Foundation website. http://www.python.org. Accessed August 16, 2017.

10. Kang Y, Engelke K. Kalender W a. A new accurate and precise 3-D segmentation method for skeletal structures in volumetric CT data. IEEE Trans Med Imaging. 2003;22:586-598.

11. Anand A, Morris MJ, Kaboteh R, et al. Analytic validation of the automated bone scan index as an imaging biomarker to standardize quantitative changes in bone scans of patients with metastatic prostate cancer. J Nucl Med. 2016; $57: 41-45$.

12. Yadav MP, Ballal S, Tripathi M, et al. ${ }^{177}$ Lu-DKFZ-PSMA-617 therapy in metastatic castration resistant prostate cancer: safety, efficacy, and quality of life assessment. Eur J Nucl Med Mol Imaging. 2017;44:81-91.

13. International Commission on Radiological Protection (ICRP). Report of the Task Group on Reference Man. ICRP publication 23. Oxford, U.K.: Pergamon Press; 1975.

14. Valentin J. Basic anatomical and physiological data for use in radiological protection: reference values: ICRP Publication 89. Ann ICRP. 2002;32:5-265.

15. Messiou C, Cook G, DeSouza NM. Imaging metastatic bone disease from carcinoma of the prostate. Br J Cancer. 2009;101:1225-1232.

16. Pyka T, Okamoto S, Dahlbender M, et al. Comparison of bone scintigraphy and ${ }^{68} \mathrm{Ga}$-PSMA PET for skeletal staging in prostate cancer. Eur J Nucl Med Mol Imaging. 2016;43:2114-2121.

17. Ulmert D, Solnes L, Thorek DL. Contemporary approaches for imaging skeletal metastasis. Bone Res. 2015;3:15024.

18. Eiber M, Maurer T, Souvatzoglou M, et al. Evaluation of hybrid ${ }^{68} \mathrm{Ga}-\mathrm{PSMA}$ ligand PET/CT in 248 patients with biochemical recurrence after radical prostatectomy. J Nucl Med. 2015;56:668-674.

19. Afshar-Oromieh A, Avtzi E, Giesel FL, et al. The diagnostic value of PET/CT imaging with the ${ }^{68} \mathrm{Ga}$-labelled PSMA ligand HBED-CC in the diagnosis of recurrent prostate cancer. Eur J Nucl Med Mol Imaging. 2015;42:197-209. 